\title{
Establishment of a novel non-alcoholic fatty liver disease model using cholesterol-fed rabbits with reference to the potential role of endoplasmic reticulum stress
}

\author{
YANLI WANG $^{1 *}$, PENG ZHANG ${ }^{2 *}$, XINGLI SU ${ }^{3}$, QI YU ${ }^{4}$, YULONG CHEN $^{4}$, \\ HUA GUAN ${ }^{4,5}$, ENQI LIU ${ }^{4,5}$ and JIANGLIN FAN ${ }^{1,6}$
}

\begin{abstract}
Departments of ${ }^{1}$ Pathology, ${ }^{2}$ Surgery, and ${ }^{3}$ Pathophysiology; ${ }^{4}$ Shaanxi Key Laboratory of Ischemic Cardiovascular Disease, Institute of Basic and Translational Medicine, Xi'an Medical University, Xi'an, Shaanxi 710021; ${ }^{5}$ Research Institute of Atherosclerotic Disease, Xi'an Jiaotong University Cardiovascular Research Center, Xi'an, Shaanxi 710061, P.R. China;

${ }^{6}$ Department of Molecular Pathology, Faculty of Medicine, Graduate School of Medical Sciences, University of Yamanashi, Yamanashi 409-3898, Japan
\end{abstract}

Received January 16, 2018; Accepted July 3, 2018

DOI: $10.3892 / \mathrm{mmr} .2018 .9258$

\begin{abstract}
The aim of the present study was to establish a non-alcoholic fatty liver disease (NAFLD) model using cholesterol-fed rabbits and to investigate whether endoplasmic reticulum stress (ERS) serves a role in the pathogenesis of NAFLD. A total of 20 male rabbits were randomly divided into 3 groups: Those fed a normal chow diet, a high cholesterol diet (HCD) or a high fat and high cholesterol diet (HFCD) for 12 weeks. Total cholesterol, triglycerides and free fatty acids of plasma and the liver were measured. At 12 weeks, a glucose tolerance test was performed. The steatosis of the liver was evaluated using hematoxylin and eosin and Oil Red $\mathrm{O}$ staining. Expression levels of glucose regulation protein 78, CCAAT/enhancer-binding protein homologous protein, c-Jun N-terminal kinase (JNK) and caspase-12 mRNA was analyzed by reverse transcription-quantitative polymerase chain reaction. Plasma levels of total cholesterol, triglycerides and free fatty acids in the HCD and HFCD groups were significantly higher when compared with those in the control group $(\mathrm{P}<0.05$ or $\mathrm{P}<0.01)$. Histological analysis revealed that HCD and HFCD groups demonstrated marked differences in the fatty liver compared with the control group, while there was no significant difference between the HCD and HFCD groups. JNK and caspase-12 expression were significantly increased in the HCD and HFCD groups when compared with the control.
\end{abstract}

Correspondence to: Professor Jianglin Fan, Department of Pathology, Xi'an Medical University, 1 Xinwang Road, Weiyang, Xi'an, Shaanxi 710021, P.R. China

E-mail: jianglinfan@hotmail.com

*Contributed equally

Key words: non-alcoholic fatty liver disease, cholesterol, hypercholesterolemia, rabbits, endoplasmic reticulum stress
The HCD and HFCD groups exhibited prominent fatty livers, a typical pathological feature of NAFLD. However, the addition of high fat levels in the cholesterol diet did not increase the severity of hepatic steatosis in HFCD when compared with the HCD group. Thus, the ERS pathway may participate in the pathogenesis of NAFLD, and cholesterol-fed rabbits may become a novel model for the study of NAFLD.

\section{Introduction}

Non-alcoholic fatty liver disease (NAFLD) is a pathological syndrome characterized by fatty liver unassociated with alcohol consumption. NAFLD unassociated with genetic and-environment-associated metabolic stress diseases, including simple non-alcoholic fatty liver (NAFL), non-alcoholic steatohepatitis (NASH) and liver cirrhosis. NAFLD is often associated with obesity, hyperglycemia, hyperlipidemia, hyperinsulinemia, hypertension and atherosclerosis (1).

With lifestyle changes in modern society, the incidence of NAFLD increases each year and shows high prevalence at young age (2). The prevalence of NAFLD in Western countries is $20-30 \%$ (3) whereas in the Asia Pacific region, the prevalence is up to $24 \%$ (4). Italian 10-year cohort study showed that the prevalence of NAFLD was increased by $2 \%$ each year (5). In China, NAFLD affects over a quarter of the general population, and its prevalence is rapidly increasing with lifestyle changes and the aging population $(6,7)$.

In spite of this, the pathogenesis of NAFLD has not yet been fully elucidated (8). In 1998, Day and James (9) proposed a hypothesis called 'secondary strike' for the pathogenesis of NAFLD. According to the hypothesis, the strike is primarily a reactive product increased of oxidative metabolism, leading to lipid peroxidation with cytokines, and mitochondrial uncoupling protein. Their study found that abnormal metabolism of fatty acids leads to generation of reactive oxygen species (ROS) (9). These functional abnormalities may lead to endoplasmic reticulum stress (ERS). It has been known that the 
endoplasmic reticulum plays an important role in both fatty acid synthesis and cholesterol metabolism (10). In addition, the endoplasmic reticulum is the site of triglyceride formation (11). The potential role of the endoplasmic reticulum in the regulation of lipid droplet number, composition, size and in lipogenesis and lipolysis remains an important but unexplored area (12). When the endoplasmic reticulum becomes stressed due to the accumulation of newly synthesized unfolded proteins, stress signaling systems are activated, including the c-Jun-NH2 terminal kinase (JNK) pathways, as well as networks activated by oxidative stress, which can influence lipid metabolism. Previous studies have demonstrated that disturbed endoplasmic reticulum homeostasis-induced apoptosis (13) and lipid accumulation (14) in the liver may contribute to the development of NAFLD (15).

Despite this, there is a lack of ideal experimental animal models for NAFLD, which limits research into its etiology, pathogenesis, and prevention. The current ob/ob/db/db mouse is the most used NAFLD model for research. The ob/ob mouse is genetically deficient in the leptin gene, while the $\mathrm{db} / \mathrm{db}$ mouse has a natural mutation in the leptin receptor gene. Normal feed can cause fatty liver, but not in the form of NASH $(16,17)$. Congenital and genetically modified models are difficult to obtain and expensive to purchase. Lack of methionine and choline diet in rats can cause NAFLD, but these diet feeding reduced muscle and fat with body weight loss, which is different from human NAFLD $(18,19)$. NAFLD rat models induced by $\mathrm{CCl} 4$ suffer from high mortality, while this model lacks obesity and IR. Compared with mice and rats, rabbits have unique features of lipid metabolism similar to those of humans $(20,21)$. Unlike mice in which high density lipoproteins (HDLs) is the predominant plasma lipoproteins, rabbit lipoprotein profiles are LDL-rich, which is similar to those of humans. Secondly, it has been reported that cholesteryl ester transfer protein (CETP) plays a central role in lipid metabolism and the atherosclerotic process. Rabbits have abundant CETP activity in the plasma as do humans, whereas mice do not have an endogenous CETP gene. In addition, like humans, rabbits do not have hepatic apoB mRNA editing activity, so rabbit apoB-48 is only present in chylomicrons. However, apoB-48 is present in all apoB-containing particles such as very low-density lipoproteins (VLDL), LDLs and chylomicrons in mice. Therefore, rabbits provide a unique system to study lipid metabolism. In the present study, we attempted to generate a rabbit NAFLD model by feeding with a diet containing high cholesterol or high cholesterol with high fat to mimic human NAFLD.

\section{Materials and methods}

Animals and diets. Japanese white rabbits were provided by the Laboratory Animal Center of Xi'an Jiaotong University. A total of 20 male rabbits (16 weeks old, about $2.0 \mathrm{~kg}$ body weight) were randomly divided into three groups: The control group $(n=4)$ was fed with a chow diet, HCD group $(n=8)$ was fed with a chow diet supplemented with $1 \%$ cholesterol and high fat and high cholesterol diet (HFCD) group $(n=8)$ was fed a chow diet containing $6.7 \%$ lard and $1 \%$ cholesterol for 12 weeks. Rabbits were fed with a restricted diet (100 g/day/animal) with free access to water.
Cholesterol was purchased from Wako Pure Chemical Industries, Ltd., (Osaka, Japan). The experimental protocols were approved by the Animal Administration Committee of Xi'an Medical University (Shaanxi, China) and performed according to the Xi'an Medical University Guidelines for Animal Experimentation and the Guide for the Care and Use of Laboratory Animals Published by the US National Institutes of Health (NIH publication no. 85-23, revised 1996).

Determination of plasma lipid levels and glucose tolerance test. Rabbits were fasted for $16 \mathrm{~h}$ prior to blood collection. Blood samples were collected via the ear artery and put into tubes containing EDTA and the plasma separated by centrifugation at 2,000 $\mathrm{rpm} / \mathrm{min}\left(20 \mathrm{~min}, 4^{\circ} \mathrm{C}\right)$. Plasma total cholesterol (TC) and triglyceride (TG) levels were measured biweekly using commercial assay kits (BioSino Bio-technology and Science, Inc., Beijing, China). At 12 weeks, blood samples were collected to measure plasma free fatty acid (FFA) levels using commercial assay kits (BioVision, Inc., Milpitas, CA, USA). At the same time, plasma alanine aminotransferase (ALT) and aspartate aminotransferase (AST) were measured using commercial assay kits (Pars Azmun, Tehran, Iran).

To evaluate the effect of high cholesterol diet (HCD) on glucose metabolism, rabbits were fasted overnight and an intravenous glucose tolerance test (IVGTT) was performed using the method as described previously (22). A bolus of glucose ( $0.6 \mathrm{~g} / \mathrm{kg}$ body weight) was injected through the ear vein and blood samples were collected through the ear artery at 5, 10, 15, 20, 30, 45, 60, 75 and $120 \mathrm{~min}$. Plasma glucose levels were measured using commercial assay kits (Wako Pure Chemical Industries, Ltd., Osaka, Japan). The incremental area under the curve (AUC) was calculated according to the trapezium rule as described previously (23).

Analysis of lipid metabolism and ERS-related gene expression of the liver. At the end of the experiment, the rabbits were euthanized by overdose of injection of sodium barbital solution. A piece of liver was quickly put into liquid nitrogen for homogenate and RNA extraction. About $100 \mathrm{mg}$ liver tissue was homogenized in $0.75 \mathrm{ml}$ of methanol and chloroform $(2: 1)$, and lipids were extracted from the chloroform fraction and N2-dried to remove the chloroform. Then, the hepatic TC and TG contents were determined using Wako kits (Wako Pure Chemical Industries, Ltd.). For the hepatic FFA assay, liver tissue was homogenized in $0.2 \mathrm{ml}$ of chloroform with $1 \%$ Triton X-100. Fatty acids were extracted in the chloroform fraction and N2-dried to remove the chloroform. Then, the hepatic tissue FFA contents were determined using a FFA quantification kit (BioVision, Mountain View, CA, USA) (24). For reverse transcription-quantitative polymerase chain reaction (RT-qPCR) reactions, total RNA was extracted from liver samples using TRIzol Plus (Invitrogen; Thermo Fisher Scientific, Inc., Waltham, MA, USA) according to manufacturer's instructions. A total of $10 \mu \mathrm{g}$ total RNA was reverse transcribed into cDNA using Takara SuperScript ${ }^{\mathrm{TM}} \mathrm{RT}$ reagent kit with gDNA eraser (cat. no. RR047A; Takara Biotechnology Co., Ltd., Dalian, China). A mixture of dNTPs were included in the PrimeScript RT enzyme Mix, and OligodT primers and random 6 mers were included in the RT primer mix of the SuperScript ${ }^{\mathrm{TM}}$ RT reagent kit. ERS-associated gene 
expression was measured by RT-qPCR. Forward and reverse primer sequences of ERS-associated gene are presented in Table II. GAPDH was used as the reference gene and the primer sequences are included in Table II. The thermocycling conditions used for qPCR were as follows: $95^{\circ} \mathrm{C}$ for $30 \mathrm{sec}$, followed by 40 cycles of $95^{\circ} \mathrm{C}$ for $5 \mathrm{sec}$ and $60^{\circ} \mathrm{C}$ for $30 \mathrm{sec}$, and then, $95^{\circ} \mathrm{C}$ for $15 \mathrm{sec}, 60^{\circ} \mathrm{C}$ for $30 \mathrm{sec}$ and $95^{\circ} \mathrm{C}$ for $15 \mathrm{sec}$. RT-qPCR analysis was performed using Takara SYBR Green kit (cat. no. DRR820A; Takara Biotechnology Co., Ltd.) by TaKaRa TP800 (Takara Bio, Inc., Otsu, Japan). Values of the quantification cycle $(\mathrm{Cq})$ were used for quantification with the $2^{-\Delta \Delta \mathrm{Cq}}$ method, as described previously (25).

Western blotting analysis. Fresh liver samples were homogenized in lysis buffer at $4^{\circ} \mathrm{C}$ followed by centrifugation at $10,000 \mathrm{rpm}$ at $4^{\circ} \mathrm{C}$ for $10 \mathrm{~min}$. The resultant supernatants were collected and subjected to western blotting. Briefly, $15 \mu \mathrm{g}$ lysates were fractionated on $10 \%$ SDS-polyacrylamide gels and then transferred to Sequi-Blot polyvinylidene fluoride membranes (Bio-Rad, Hercules, CA, USA). The membranes were incubated with each primary antibody $(\mathrm{Ab})(1: 1,000)$ at $4^{\circ} \mathrm{C}$ overnight, as recommended in the manufacturer's instructions. After washing 3 times, they were incubated with horseradish peroxidase conjugated secondary $\mathrm{Ab}$ for $2 \mathrm{~h}$. Signals were detected using the Immobilon reagent (Millipore, Billerica, MA, USA) and visualized using an LAS-400 Lumino Image Analyzer (Fujifilm, Co., Tokyo, Japan) (26). Primary Abs of CHOP (L63F7), JNK (9251L) and Caspase-12 (2202P) were purchased from Cell Signaling Technology, Inc., (Danvers, MA, USA). Anti-GRP78 (ab89789) was purchased from Abcam (Cambridge, UK).

Pathological analysis. At the end of the experiment, a piece of liver was stocked in liquid nitrogen and embedded in optimal cutting temperature (OCT) compound and cut in $8-\mu \mathrm{m}$ sections followed by oil red $\mathrm{O}$ staining. The areas stained by oil red $\mathrm{O}$ were quantified to assess the areas of connective tissue and lipid deposition, respectively, using an image analysis system (WinROOF v6.5) $(24,27)$. At the same time, formalin-fixed liver was embedded in paraffin and cut in $4-\mu \mathrm{m}$ thick sections and stained with hematoxylin and eosin (HE).

Statistical analysis. Data are expressed as the mean \pm standard error of the mean. Statistical analysis was performed using analysis of variance and Dunnett's post hoc test, and the data with an equal $F$ value, using GraphPad Prism 5 software (GraphPad Software, Inc., La Jolla, CA, USA). P<0.05 was considered to indicate a statistically significant difference.

\section{Results}

Plasma lipid levels, liver function and glucose tolerance test. Plasma TC levels were remarkably increased in both HCD and HFCD groups compared with control group $(\mathrm{P}<0.05$ or $\mathrm{P}<0.01)$. Plasma TG significantly increased in HFCD group compared with control group $(\mathrm{P}<0.05)$. At the end of the experiment, plasma FFA was measured and the results showed that FFA was significantly increased in HCD and HFCD groups compared with control group $(\mathrm{P}<0.05)$. We also performed a glucose tolerance test and found that glucose
Table I. Test results of liver function.

\begin{tabular}{lcc}
\hline Group & ALT (U/l) & AST (U/l) \\
\hline Control & $43 \pm 10$ & $40 \pm 12$ \\
HCD & $46 \pm 11$ & $81 \pm 13$ \\
HFCD & $42 \pm 7$ & $78 \pm 9$ \\
\hline
\end{tabular}

The results are presented as the mean \pm standard deviation (control, $\mathrm{n}=4$; HCD, $\mathrm{n}=8$; HFCD, $\mathrm{n}=8$ ). ALT, alanine aminotransferase; AST, aspartate aminotransferase; $\mathrm{HCD}$, high cholesterol diet; HFCD, high fat and high cholesterol diet.

Table II.Primer sequences for reverse transcription-quantitative polymerase chain reaction.

\begin{tabular}{ll}
\hline Genes & \multicolumn{1}{c}{ Primer sequence (5'-3') } \\
\hline GRP78 & F: TGGCTACTCCTCTCTGAACAAC \\
GRP78 & R: CGAGAATCATGGCGGAGATCT \\
JNK & F: CACCACCAAAGATTCCTGACA \\
JNK & R: GGCTGCCCTCGTATAACTCC \\
CHOP & F: AGAACTGGGACGAAGAGACC \\
CHOP & R: AGCTAGCTGTGCCACTTTCC \\
Caspase-12 & F: GGAAGGTAGGCAAGACTGGTT \\
Caspase-12 & R: TCAATAGTGGGCATCTGGGTC \\
GAPDH & F: CCACTTTGTGAAGCTCATTTCCT \\
GAPDH & R: TCGTCCTCCTCTGGTGCTCT
\end{tabular}

GRP78, glucose regulation protein 78; CHOP, CCAAT/enhancerBinding protein homologous protein; JNK, c-Jun N-terminal kinases; $\mathrm{F}$, forward; R, reverse.

clearance was delayed in HCD and HFCD groups compared with control group $(\mathrm{P}<0.05$ or $\mathrm{P}<0.01 ;$ Fig. 1$)$. Plasma ALT and AST levels were not remarkably different among the three groups (Table I).

Pathological analysis of liver. Compared with the liver of the control group, the surface of the liver of HCD and HFCD groups appeared obviously yellow and greasy (Fig. 2, left). Body weight and the ratio of the liver weight to body weight were not statistically significant in the three groups (Fig. 2A and B).

The analysis of liver histology revealed that there were a large number of vacuoles in the hepatocytes of the HCD and HFCD groups compared with control group as demonstrated by both $\mathrm{HE}$ and oil red $\mathrm{O}$ staining (Fig. 3). The areas stained by oil red $\mathrm{O}$ were measured to evaluate the severity of connective tissue and lipid deposition as previously described $(24,26)$ and the results showed that NAFLD model was successfully established in both HCD and HFCD groups even though liver histological features were not significantly different (Fig. 3). To evaluate lipid compositions of liver, TC, TG and FFA contents were measured using liver homogenate. The results showed that hepatic TC and FFA contents were significantly increased in HCD and HFCD groups compared with control 


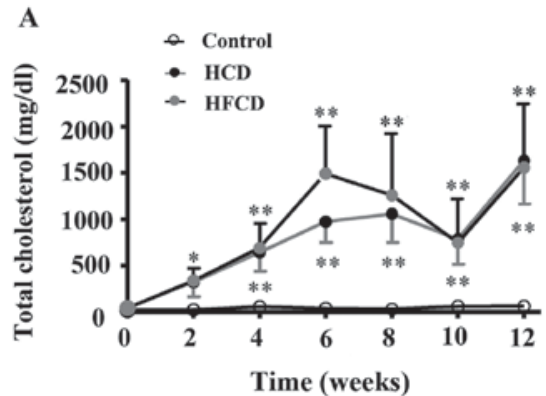

B

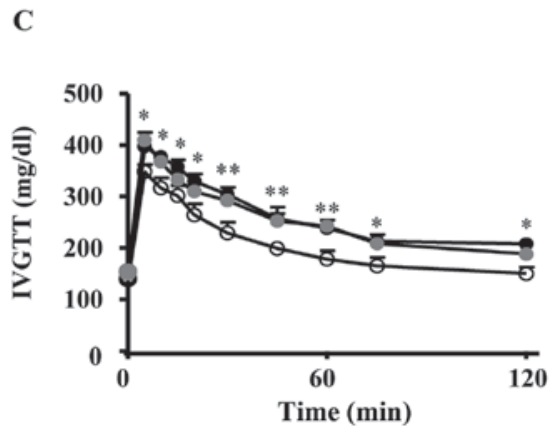

Figure 1. Plasma lipid levels and glucose tolerance test. (A) Plasma total cholesterol was notably increased in HCD and HFCD groups when compared with the control group. (B) Plasma triglyceride was significantly increased in the HFCD group when compared with HCD and control groups. (C) Plasma glucose tolerance test revealed that glucose clearance was delayed in the HCD and HFCD groups when compared with the control group. $n=4$ for control, $n=8$ for HCD and HFCD. " $\mathrm{P}<0.05$ and ${ }^{* *} \mathrm{P}<0.01$ vs. control. HCD, high cholesterol diet; HFCD, high fat and high cholesterol diet; IVGTT, intravenous glucose tolerance test.
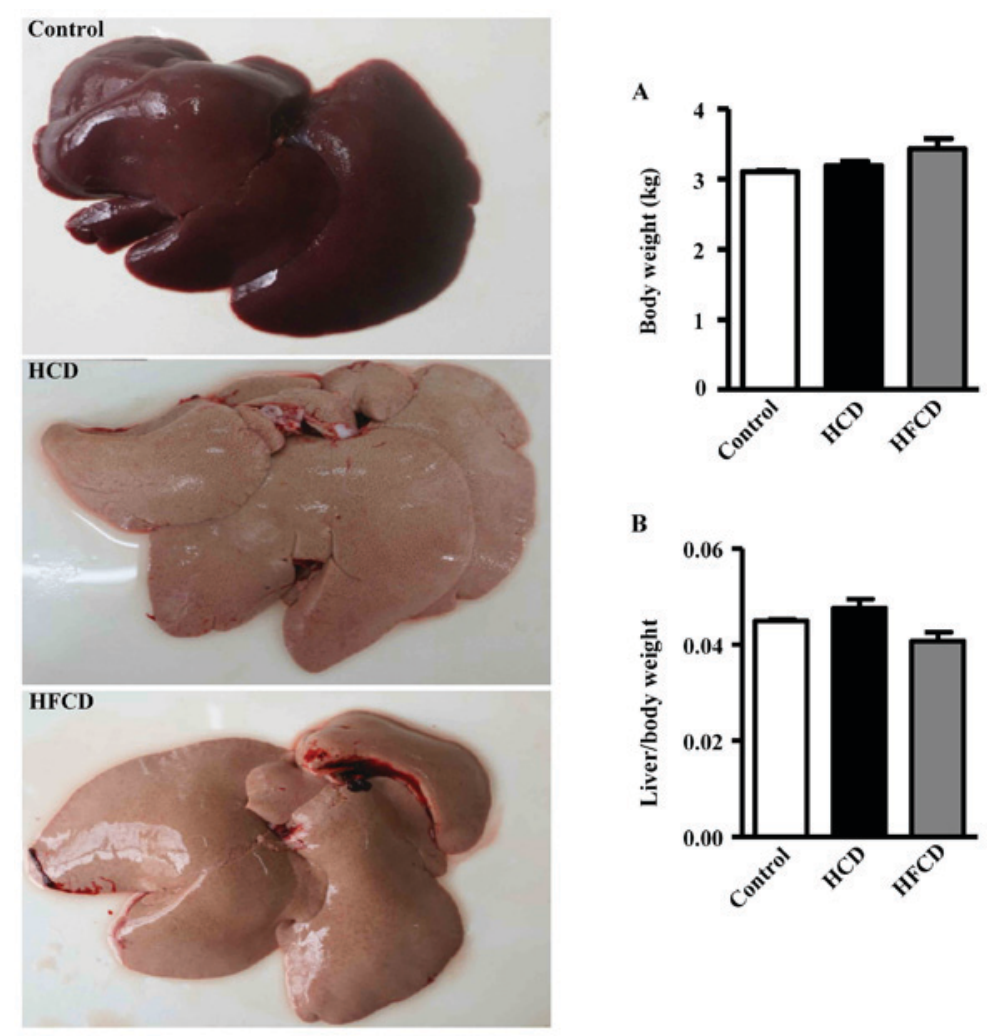

B

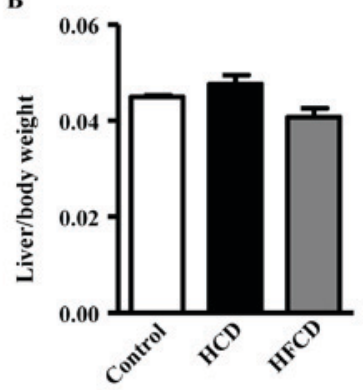

Figure 2. Morphology of liver and comparison of liver weight. Livers from the control group were dark red; however, those of the HCD and HFCD groups were yellow and greasy (left-hand panel). (A) Body weight and (B) the ratio of the liver weight to body weight were not statistically significant among the three groups. $\mathrm{n}=4$ for control, $\mathrm{n}=8$ for HCD and HFCD. HCD, high cholesterol diet; HFCD, high fat and high cholesterol diet.

group, and hepatic TG content was significantly increased in HFCD group compared with control group. However significant differences were not observed between the HCD and HFCD groups $(\mathrm{P}<0.05$ or $\mathrm{P}<0.01$; Fig. 4).
ERS indicators. Expression levels of ERS-associated liver genes were compared by RT-qPCR to investigate whether ERS plays any role in the pathogenesis of NAFLD. The genes and primer sequence were showed in Table II. Glucose regulation 

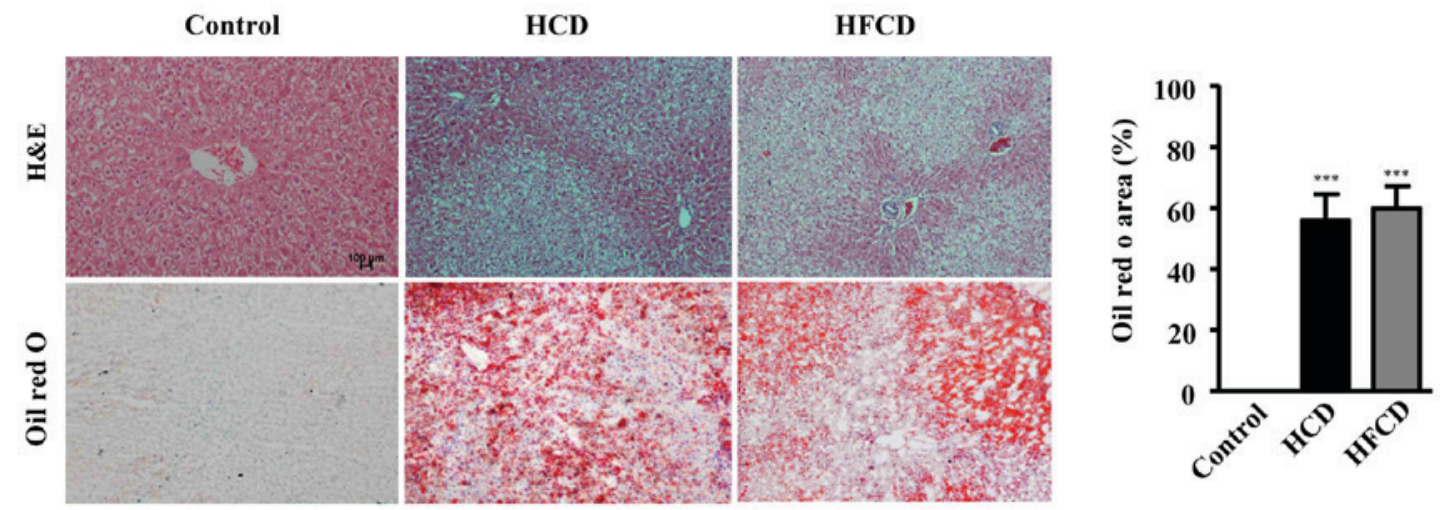

Figure 3. Analysis of liver histology. The liver sections were stained with HE. There were many vacuoles in the HCD and HFCD groups, but not in control group. A larger number of red stained lipid droplets were observed in the HCD and HFCD groups with Oil Red O staining (left-hand panel). Scale bars, $100 \mu \mathrm{m}$; magnification, $\mathrm{x} 20$. The positive area of Oil Red O staining was significantly increased in the HCD and HFCD groups when compared with the control group (right-hand panel). $\mathrm{n}=4$ for control, $\mathrm{n}=8$ for HCD and HFCD. ${ }^{* * *} \mathrm{P}<0.001$ vs. control. HCD, high cholesterol diet; HFCD, high fat and high cholesterol diet; HE, hematoxylin and eosin.

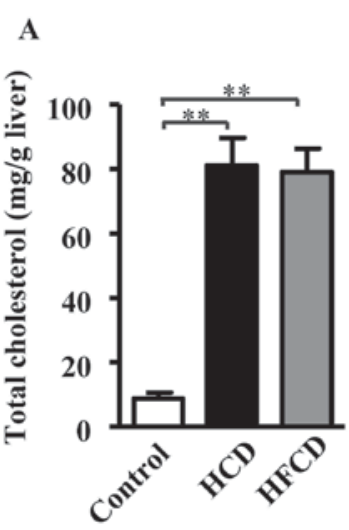

C

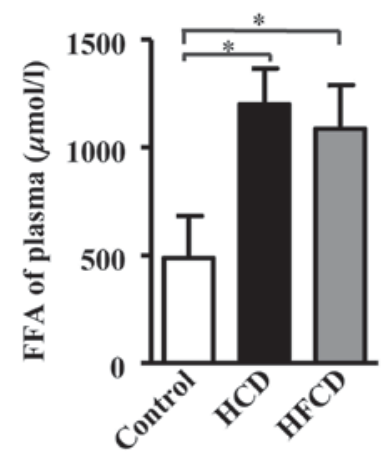

B

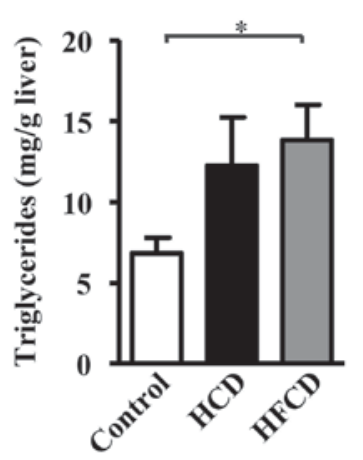

D

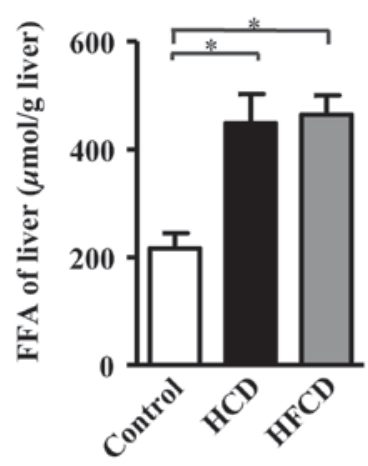

Figure 4. Determination of lipid compositions of the liver. (A) Total cholesterol significantly increased in the HCD and HFCD groups compared with the control group. (B) Triglyceride significantly increased in the HFCD group compared with the control group. (C) FFA levels of liver tissue homogenate and (D) plasma were significantly increased in the HCD and HFCD groups when compared with the control group. ${ }^{*} \mathrm{P}<0.05$ and ${ }^{* *} \mathrm{P}<0.01$, as indicated. $\mathrm{n}=4$ for control, $\mathrm{n}=8$ for HCD and HFCD. HCD, high cholesterol diet; HFCD, high fat and high cholesterol diet; FFA, free fatty acid.

protein 78 (GRP78) mRNA expression was significantly decreased in the HCD and HFCD groups compared with the control group. JNK mRNA expression was increased in HCD group compared with control and HFCD group, while there was no significant difference between HFCD and control groups. Caspase-12 mRNA expression was also increased

in the HCD and HFCD groups compared with control, and there was a significant difference between the HCD and HFCD groups $(\mathrm{P}<0.05$ or $\mathrm{P}<0.01)$. CCAAT/enhancer-binding protein homologous protein (CHOP) mRNA expression were not statistically significant in the three groups. The protein expression levels of the ERS indicators were measured and the results exhibited a similar trend to the mRNA expression levels (Fig. 5).

\section{Discussion}

At present, NAFLD has become a serious challenges for human health. Lack of an ideal animal models hampers the study of NAFLD mechanisms and development of therapeutics. Based on the advantages of rabbit lipid metabolism features being similar to humans, this experiment established the NAFLD model using cholesterol-fed JW rabbits. Two types of diets were used and both HCD and HFCD could induce NAFLD features in rabbits. The analysis of liver histology showed that HCD and HFCD groups were not significantly different in terms of hepatic steatosis. This result suggested that HCD is the major factor for inducing NAFLD in rabbit model, and a high fat in the diet is unable to accelerate the formation of NAFLD in a rabbit model. Therefore, high cholesterol is a key factor in establishing NAFLD in rabbits.

TC, TG and FFA levels of liver tissue homogenate were significantly increased in HCD and HFCD groups compared with the control group. This result revealed that there was accumulation of excessive cholesterol and other lipids in the liver compatible with the observation that a large number of vacuoles or oil red $\mathrm{O}$ stained lipid droplets were found in the livers of HCD and HFCD groups even though no difference was observed between the HCD and HFCD groups.

The endoplasmic reticulum plays an important role in lipid metabolism. When a large amount of lipid accumulates in the endoplasmic reticulum, stress signaling systems may be activated thus influencing lipid metabolism. Previous studies have found that fatty acid metabolism in the endoplasmic reticulum will weaken cellular autophagy functions (28), producing a certain amount of ROS (9). In the early stage of ERS, expression of endoplasmic reticulum molecular 
A

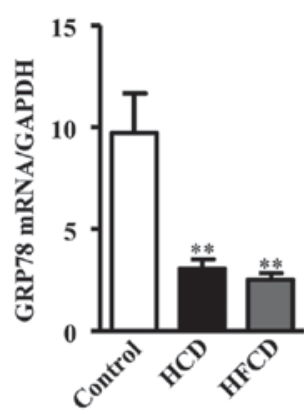

C

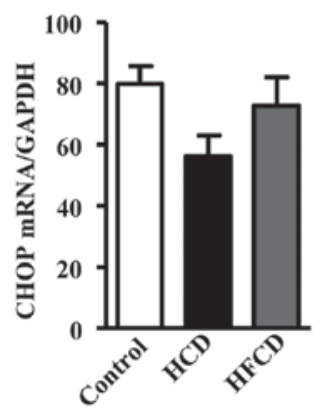

B

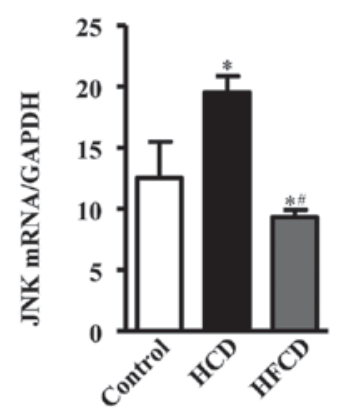

D

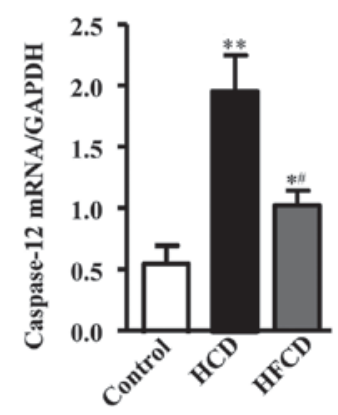

$\mathbf{E}$

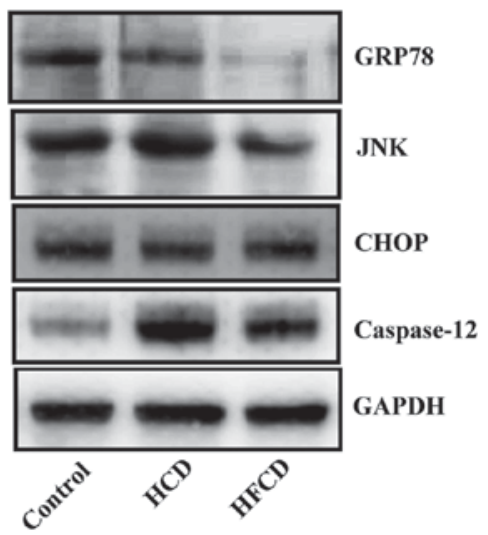

Figure 5. Analysis of ERS indicators in liver. (A) GRP78 mRNA expression was significantly decreased in the HCD and HFCD groups when compared with the control group. (B) JNK expression was markedly increased in the HCD group when compared with the control and HFCD groups, while there was no significant difference between the HFCD and control groups. (C) CHOP expression was not statistically significant among the three groups. (D) Caspase-12 levels were significantly increased in the HCD group when compared with the control and HFCD groups. ${ }^{*} \mathrm{P}<0.05$ and ${ }^{* *} \mathrm{P}<0.01$ vs. control; ${ }^{*} \mathrm{P}<0.05$ vs. $\mathrm{HCD}$. $\mathrm{n}=4$ for control, $\mathrm{n}=8$ for HCD and HFCD. (E) Protein expression of ERS indicators were detected in the liver, and the protein expression trends were similar to those observed for gene expression ( $\mathrm{n}=3$ ). ERS, endoplasmic reticulum stress; HCD, high cholesterol diet; HFCD, high fat and high cholesterol diet; GRP78, glucose regulation protein 78; CHOP, CCAAT/enhancer-binding protein homologous protein; JNK, c-Jun N-terminal kinase.

chaperone protein and GRP78 will increase and assist cell deal with unfolded protein. However, prolonged ERS can cause apoptosis (5), the cell apoptosis process was mediated by ROS and ERS (9). The cell apoptosis caused by ERS is called the endoplasmic reticulum associated death (ERAD) pathway. The ERAD pathway consists of ERS induced CHOP which activates the JNK pathway and/or caspase-12 proteolytic enzyme (29). In our study, we found that GRP78 mRNA expression was significantly decreased in both HCD and HFCD groups. Prolonged ERS induced by lipid accumulation can cause hepatocyte apoptosis. Therefore, with the increase in cell death, GRP78 expression may be decreased accordingly. JNK and caspase-12 expression levels were notably increased in the HCD group. Caspase-12 expression was notably increased in the HFCD group while CHOP expression differences were not statistically significant among the three groups. In the current study, the results suggested that the cell apoptosis may have been caused by the activation of the JNK and caspase-12 pathways rather than the CHOP pathway, which may determine the severity of hepatic steatosis and apoptosis. These results suggest that accumulation of lipids in hepatocytes may lead to ERS by which hepatocyte apoptosis may be enhanced although this hypothesis remains to be verified in the future.

\section{Acknowledgements}

Not applicable.

\section{Funding}

The present study was supported in part by a research grant from Shaanxi Science and Technology Hall Fund Project of China (grant nos. 2016SF-107, 2017JZ028 and 17JS116), Research Initiation Funds for Doctors of Xi'an Medical University (grant no. 2016DOC25) and the National Natural Science Foundation of China (grant nos. 81770457 and 81773795).

\section{Availability of data and materials}

All data generated or analyzed during this study are included in this published article.

\section{Authors' contributions}

YW and PZ performed the administration and feed of experimental animals, and analyzed plasma lipids levels and the gene expression of ERS indicators in liver. XS performed the glucose tolerance test and analyzed the data. QY performed the histological examination of the liver and analyzed the data. YC and HG analyzed the protein expression of ERS indicators in the liver and revised the figures. EL contributed to the experimental design and drafted the manuscript. JF was a major contributor in designing the experiment, revising the manuscript critically for important intellectual content and gave final approval of the version to be published. All authors read and approved the final manuscript. 


\section{Ethics approval and consent to participate}

The experimental protocols were approved by the Animal Administration Committee of Xi'an Medical University (Shaanxi, China) and performed according to the Xi'an Medical University Guidelines for Animal Experimentation and the Guide for the Care and Use of Laboratory Animals Published by the US National Institutes of Health (NIH Publication no. 85-23, revised 1996).

\section{Patient consent for publication}

Not applicable.

\section{Competing interests}

The authors declare that they have no competing interests.

\section{References}

1. DeFilippis AP, Blaha MJ, Martin SS, Reed RM, Jones SR, Nasir K, Blumenthal RS and Budoff MJ: Nonalcoholic fatty liver disease and serum lipoproteins: The multi-ethnic study of atherosclerosis. Atherosclerosis 227: 429-436, 2013.

2. Kwak JH, Jun DW, Lee SM, Cho YK, Lee KN, Lee HL, Lee OY, Choi HS and Yoon BC: Lifestyle predictors of obese and non-obese patients with nonalcoholic fatty liver disease: A cross-sectional study. Clin Nutr pii: S0261-5614: 30301, 2017.

3. Sayiner M, Koenig A, Henry L and Younossi ZM: Epidemiology of nonalcoholic fatty liver disease and nonalcoholic steatohepatitis in the United States and the rest of the world. Clin Liver Dis 20: 205-214, 2016.

4. Chitturi S, Farrell GC, Hashimoto E, Saibara T, Lau GK and Sollano JD; Asia-pacific working party on NAFLD: Non-alcoholic fatty liver disease in the Asia-Pacific region: Definitions and overview of proposed guidelines. J Gastroenterol Hepatol 22: 778-787, 2007.

5. Bedogni G, Miglioli L, Masutti F, Tiribelli C, Marchesini G and Bellentani S: Prevalence of and risk factors for nonalcoholic fatty liver disease: The Dionysos nutrition and liver study. Hepatology 42: 44-52, 2005.

6. Dai H, Wang W, Chen R, Chen Z, Lu Y and Yuan H: Lipid accumulation product is a powerful tool to predict non-alcoholic fatty liver disease in Chinese adults. Nutr Metab (Lond) 14: 49, 2017.

7. Li Z, Xue J, Chen P, Chen L, Yan S and Liu L: Prevalence of nonalcoholic fatty liver disease in mainland of China: A meta-analysis of published studies. J Gastroenterol Hepatol 29: 42-51, 2014.

8. Cheung $\mathrm{O}$ and Sanyal AJ: Recent advances in nonalcoholic fatty liver disease. Curr Opin Gastroenterol 25: 230-237, 2009.

9. Day C and James O: Steatohepatitis: A tale of two 'hits'? Gastroenterology 114: 842-845, 1998.

10. Hotamisligil GS: Endoplasmic reticulum stress and the inflammatory basis of metabolic disease. Cell 140: 900-917, 2010.

11. Wolins NE, Brasaemle DL and Bickel PE: A proposed model of fat packaging by exchangeable lipid droplet proteins. FEBS Lett 580: 5484-5491, 2006.
12. Gregor MG and Hotamisligil GS: Thematic review series: Adipocyte Biology. Adipocyte stress: The endoplasmic reticulum and metabolic disease. J Lipid Res 48: 1905-1914, 2007.

13. Wei Y, Wang D, Topczewski F and Pagliassotti MJ: Saturated fatty acids induce endoplasmic reticulum stress and apoptosis independently of ceramide in liver cells. Am J Physiol Endocrinol Metab 291: E275-E281, 2006.

14. Werstuck GH, Lentz SR, Dayal S, Hossain GS, Sood SK Shi YY, Zhou J, Maeda N, Krisans SK, Malinow MR and Austin RC: Homocysteine-induced endoplasmic reticulum stress causes dysregulation of the cholesterol and triglyceride biosynthetic pathways. J Clin Invest 107: 1263-1273, 2001.

15. Jung TW, Kim HC, Abd El-Aty AM and Jeong JH: Maresin 1 attenuates NAFLD by suppression of endoplasmic reticulum stress via AMPK-SERCA2b pathway. J Biol Chem 293: 3981-3988, 2018.

16. Catmiel-Haggai M, Cederbaum AI and Nieto N: A high-fat diet leads to the progression of non-alcoholic fatty liver disease in obese rats. FASEB J 19: 136-138, 2005.

17. Wortham M, He L, Gyamfi M, Copple BL and Wan YJ: The transition from fatty liver to NASH associates with SAMe depletion in $\mathrm{db} / \mathrm{db}$ mice fed a methionine choline-deficient diet. Dig Dis Sci 53: 2761-2774, 2008.

18. Diehl AM: Lessons from animal models of NASH. Hepatol Res 33: 138-144, 2005.

19. London RM and Geroge J: Pathogenesis of NASH: Animal models. Clin Liver Dis 11: 55-74, viii, 2007.

20. Fan J, Kitajima S, Watanabe T, Xu J, Zhang J, Liu E and Chen YE: Rabbit models for the study of human atherosclerosis: From pathophysiological mechanisms to translational medicine. Pharmacol Ther 146: 104-119, 2015.

21. Fan $\mathrm{J}$ and Watanabe T: Transgenic rabbits as therapeutic protein bioreactors and human disease models. Pharmacol Ther 99: 261-282, 2003.

22. Zhao S, Zhang C, Lin Y, Yang P, Yu Q, Chu Y, Yang P, Fan J and Liu E: The effects of rosiglitazone on aortic atherosclerosis of cholesterol-fed rabbits. Thromb Res 123: 281-287, 2008

23. Liu E, Kitajima S, Higaki Y, Morimoto M, Sun H, Watanabe T, Yamada N and Fan J: High lipoprotein lipase activity increases insulin sensitivity in transgenic rabbits. Metabolism 54: 132-138, 2005.

24. Ogawa T, Fujii H, Yoshizato K and Kawada N: A human-type nonalcoholic steatohepatitis model with advanced fibrosis in rabbits. Am J Pathol 177: 153-165, 2010

25. Livak KJ and Schmittgen TD: Analysis of relative gene expression data using real-time quantitative PCR and the 2(-Delta Delta C(T)) method. Methods 25: 402-408, 2001.

26. Guan H, Lin Y, Bai L, An Y, Shang J, Wang Z, Zhao S, Fan J and Liu E: Dietary cocoa powder improves hyperlipidemia and reduces atherosclerosis in apoE deficient mice through the inhibition of hepatic endoplasmic reticulum stress. Mediators Inflamm 2016: 1937572, 2016.

27. Hübscher SG: Histological assessment of non-alcoholic fatty liver disease. Histopathology 49: 450-465, 2006

28. Deng X, Pan X, Cheng C, Liu B, Zhang H, Zhang Y and $\mathrm{Xu} \mathrm{K}$ : Regulation of SREBP-2 intracellular trafficking improves impaired autophagic flux and alleviates endoplasmic reticulum stress in NAFLD. Biochim Biophys Acta 1862: 337-350, 2017.

29. Zhao L and Ackerman SL: Endoplasmic reticulum stress in health and disease. Curr Opin Cell Biol 18: 444-452, 2006.

This work is licensed under a Creative Commons Attribution-NonCommercial-NoDerivatives 4.0 International (CC BY-NC-ND 4.0) License. 